\title{
sciendo
}

\section{Silencing of NAMPT leads to up-regulation of insulin receptor substrate 1 gene expression in U87 glioma cells}

\author{
Daria O. Tsymbal ${ }^{1}$, Dmytro O. Minchenko ${ }^{1,2}$, Olena Y. Luzina ${ }^{1}$, Olena O. Riabovol ${ }^{1}$, \\ Serhiy V. DANilovsk Y ${ }^{1}$, Oleksandr H. Minchenko ${ }^{1}$ \\ ${ }^{1}$ Palladin Institute of Biochemistry, National Academy of Sciences of Ukraine, Kyiv, Ukraine; \\ ${ }^{2}$ National Bohomolets Medical University, Kyiv, Ukraine \\ E-mail:ominchenko@yahoo.com
}

Objective. The aim of the present study was to investigate the effect of adipokine NAMPT (nicotinamide phosphoribosyltransferase) silencing on the expression of genes encoding IRS1 (insulin receptor substrate 1) and some other proliferation related proteins in U87 glioma cells for evaluation of the possible significance of this adipokine in intergenic interactions.

Methods. The silencing of NAMPT mRNA was introduced by NAMPT specific siRNA. The expression level of NAMPT, IGFBP3, IRS1, HK2, PER2, CLU, BNIP3, TPD52, GADD45A, and MKI67 genes was studied in U87 glioma cells by quantitative polymerase chain reaction. Antivisfatin antibody was used for detection of NAMPT protein by Western-blot analysis.

Results. It was shown that the silencing of NAMPT mRNA led to a strong down-regulation of NAMPT protein and significant modification of the expression of IRS1, IGFBP3, CLU, HK2, BNIP3, and MKI67 genes in glioma cells and a strong up-regulation of IGFBP3 and IRS1 and down-regulation of CLU, BNIP3, HK2, and MKI67 gene expressions. At the same time, no significant changes were detected in the expression of GADD45A, PER2, and TPD52 genes in glioma cells treated by siRNA specific to NAMPT. Furthermore, the silencing of NAMPT mRNA suppressed the glioma cell proliferation.

Conclusions. Results of this investigation demonstrated that silencing of NAMPT mRNA with corresponding down-regulation of NAMPT protein and suppression of the glioma cell proliferation affected the expression of IRS1 gene as well as many other genes encoding the proliferation related proteins. It is possible that dysregulation of most of the studied genes in glioma cells after silencing of NAMPT is reflected by a complex of intergenic interactions and that NAMPT is an important factor for genome stability and regulatory mechanisms contributing to the control of glioma cell metabolism and proliferation.

Key words: silencing NAMPT, mRNA expression, IRS1, IGFBP3, PER2, CLU, BNIP3, TPD52, GADD45A, U87 glioma cells

The nicotinamide phosphoribosyltransferase (NAMPT) also known as visfatin and PBEF (pre-Bcell colony-enhancing factor) is an insulin-mimetic adipokine with anti-diabetic properties, which is involved in many important biological processes, including metabolism, stress response, insulin resistance, and tumorigenesis (Flehmig et al. 2014;
Olszanecka-Glinianowicz et al. 2014; Minchenko et al. 2015a; Carbone et al. 2017; Hesari et al. 2018; Annie et al. 2019; Meram et al. 2019). This protein catalyzes the condensation of nicotinamide with 5-phosphoribosyl-1-pyrophosphate yielding to nicotinamide mononucleotide, the rate limiting component in the mammalian NAD biosynthesis pathway. It is secreted

Corresponding author: Oleksandr H. Minchenko, Prof., Department of Molecular Biology, Palladin Institute of Biochemistry, National Academy of Sciences of Ukraine, Leontovycha 9, Kyiv 01030, Ukraine; e-mail: ominchenko@yahoo.com. 
form of the adipokine and its circulating levels has been closely correlated with the white adipose tissue accumulation, obesity and contribute to metabolic and vascular complications (Flehmig et al. 2014; Li et al. 2017; Huang et al. 2018). The secreted form of NAMPT behaves both as a cytokine with immunomodulating properties and an adipokine with antidiabetic properties preferentially independent on its enzymatic activity (Zhang et al. 2017; Tabassum et al. 2018). Furthermore, there are data indicating that NAMPT-mediated NAD ${ }^{+}$biosynthesis is indispensable for the adipose tissue plasticity, systemic glucose homeostasis, and the pathological progression of obesity (Nielsen et al. 2018).

NAMPT is a pleiotropic protein implicated in the pathogenesis of cancer and obesity and its complications, including insulin resistance, diabetes, and aging (Goktas et al. 2013; Shackelford et al. 2013; Zhang et al. 2017; Zhao et al. 2017; Liang et al. 2018; Poljsak 2018; Ohanna et al. 2018). There are data reporting that inhibition of NAMPT by specific inhibitors decreases cancer growth, induces apoptosis, suppresses cell growth, and enhances the susceptibility to oxidative stress and also sensitizes the glioblastoma cells to temozolomide (Abu Aboud et al. 2016; Feng et al. 2016; Alaee et al. 2017; Xu et al. 2017; Tabassum et al. 2018). It has also been shown that down-regulation of NAMPT expression by miR-206 reduces survival of the breast cancer cells that NAMPT expression is up-regulated in pediatric gliomas and that its overexpression induces glioma and other cancers growth (Minchenko et al. 2015a; Lucena-Cacace et al. 2017; Zhao et al. 2017; Hesari et al. 2018). Moreover, NAMPT can promote hepatocellular carcinoma cell migration through the up-regulation of miR-21 (Liang et al. 2018).

NAMPT is involved in basic key cellular functions such as transcription, translation, cell signaling, and fundamental metabolism (Xu et al. 2017; Cheleschi et al. 2018; Liang et al. 2018; Nielsen et al. 2018; Shi et al. 2018). However, the underlying molecular mechanisms of NAMPT in physiological and pathological processes are not fully understood. The aim of the present study was to investigate the effect of adipokine NAMPT (nicotinamide phosphoribosyltransferase) silencing on the expression of genes encoding insulin receptor substrate 1 and some other proliferation related proteins in U87 glioma cells for revealing the possible significance of this adipokine in the intergenic interactions. Among others, insulin-like growth factor binding protein 3 (IGFBP3), hexokinase 2 (HK2), period circadian regulator 2 (PER2), clusterin (CLU), BCL2/adenovirus E1B 19 kDa interacting protein 3 (BNIP3), tumor protein D52 (TPD52), growth arrest and DNA-damage-inducible, alpha (GADD45A), and marker of proliferation Ki-67 (MKI67) have been studied. These polyfunctional proteins play an important role in the regulation of metabolism, cell proliferation, surviving and apoptosis and biological clock (Fan et al. 2018; Liu et al. 2018; Qu et al. 2018; Xiong et al. 2018; Xu et al. 2018; Zhou et al. 2018; Chen et al. 2014, 2019; Niu et al. 2019; Li et al. 2019; Wu et al. 2019).

In the present study, we provide experimental evidence that the silencing of NAMPT mRNA resulted in the down-regulation of NAMPT protein and the suppression of the glioma cell proliferation and affected the expression of IRS1 gene and many other genes encoding the proliferation related proteins, which may contribute to the control of the glioma cell metabolism and proliferation.

\section{Materials and methods}

Cell line and culture conditions. The glioma cell line U87 was obtained from ATCC (USA) and grown in high glucose $(4.5 \mathrm{~g} / \mathrm{l})$ Dulbecco's modified Eagle's minimum essential medium (DMEM, Gibco, Invitrogen, Carlsbad, CA, USA) supplemented with glutamine $(2 \mathrm{mM}), 10 \%$ fetal bovine serum (FBS, Equitech-Bio, Inc., USA), penicillin (100 units/ml; Gibco) and streptomycin (0.1 mg/ml; Gibco) at $37^{\circ} \mathrm{C}$ in incubator with $5 \% \mathrm{CO}_{2}$.

The silencing of NAMPT mRNA was introduced by NAMPT specific siRNA (PBEF siRNA (h), sc-45843, Santa Cruz Biotechnology, Inc., Dallas, TX, U.S.A.). Control siRNA-A (sc-37007, Santa Cruz Biotechnology, Inc.) was used for transfection of the control glioma cells. The transfection was performed by lipofectamine RNAiMAX for 48 h (Invitrogen, Carlsbad, CA, USA) in accordance with the manufacturer's protocol, with siRNA at a final concentration of $100 \mathrm{nM}$.

RNA isolation. Total RNA was extracted from the glioma cells using the Trizol reagent according to manufacturer's protocol (Invitrogen). The RNA pellets were washed with $75 \%$ ethanol and dissolved in nuclease-free water. For additional purification, the RNA samples were re-precipitated with 95\% ethanol and re-dissolved again in nuclease-free water. The RNA concentration and the spectral characteristics were measured using NanoDrop Spectrophotometer ND1000 (PEQLAB, Biotechnologie GmbH).

Reverse transcription and quantitative PCR analysis. The effect of NAMPT silencing on the expression levels of NAMPT, IGFBP3, IRS1, HK2, 
PER2, CLU, BNIP3, TPD52, GADD45A, and MKI67 mRNAs as well as ACTB (beta-actin) mRNA was measured in control U87 glioma cells and cells with a deficiency of NAMPT, introduced by NAMPT siRNA, by quantitative polymerase chain reaction using SYBRGreen Mix (ABgene, Thermo Fisher Scientific, Epsom, Surrey, UK), and qPCR "QuantStudio 5 Real-Time PCR System” (Applied Biosystems, USA). Thermo Scientific Verso cDNA Synthesis Kit (Germany) was used for reverse transcription. The polymerase chain reaction was performed in triplicate. The expression of beta-actin mRNA was used as control of analyzed RNA quantity. The pair of primers specific for each of the studied gene was received from Sigma-Aldrich (St. Louis, MO, U.S.A.) and used for quantitative polymerase chain reaction (Table 1).

Quantitative PCR analysis was performed using a special computer program "Differential expression calculator" and statistical analysis using Excel program and OriginPro 7.5 software as described previously (Minchenko et al. 2015b). Comparison of two means was performed by the use of two-tailed Student's t-test. $\mathrm{p}<0.05$ was considered significant in all cases. The values of NAMPT, IGFBP3, IRS1, HK2, PER2, CLU, BNIP3, TPD52, GADD45A, and MKI67 gene expressions were normalized to the expression of beta-actin mRNA and represent as percent of control (100\%). All values are expressed as mean \pm SEM from triplicate measurements performed in 4 independent experiments. The amplified DNA fragments were also analyzed on a $2 \%$ agarose gel and that visualized by SYBR* Safe DNA Gel Stain (Life Technologies, Carlsbad, CA, USA).

Western blot analysis. Cell extracts were prepared using buffers A, as previously described (Armstead et al. 1997). The proteins were resolved using sodium dodecyl sulfate-polyacrylamide gel (10\% acrylamide) electrophoresis and transferred to a polyvinylidene difluoride membrane (Immobilon-P Transfer Membrane; Millipore, Chelmsford, MA, U.S.A.) by a semi-dry blotting system. The excess sites on the membrane were saturated with $5 \%$ non-fat dried milk in TPBS (PBS containing 0.1\% Tween 20). Western blot analysis was carried out as described previously (Minchenko et al. 2004). For detection of NAMPT protein, a rabbit anti-Visfatin, PBEF (412-431) IgG

Table 1

Characteristics of the primers used for quantitative real-time polymerase chain reaction.

\begin{tabular}{|c|c|c|c|c|}
\hline Gene symbol & Gene name & Primer's sequence & $\begin{array}{l}\text { Nucleotide numbers } \\
\text { in sequence }\end{array}$ & $\begin{array}{c}\text { GenBank accession } \\
\text { number }\end{array}$ \\
\hline $\begin{array}{l}\text { NAMPT } \\
\text { (PBEF) }\end{array}$ & $\begin{array}{l}\text { Nicotinamide } \\
\text { phosphoribosyltransferase } \\
\text { (pre-B-cell colony-enhancing } \\
\text { factor; visfatin) }\end{array}$ & $\begin{array}{l}\text { F: 5'- tcttcacggtggaaaacaca } \\
\text { R: 5'- gctcctatgccagcagtctc }\end{array}$ & $700-719931-912$ & NM_005746 \\
\hline $1 R S 1$ & Insulin receptor substrate 1 & $\begin{array}{l}\text { F: 5'- agtcccagcaccaacagaac } \\
\text { R: 5'- tcatccgaggagatgaaacc }\end{array}$ & $1094-11131341-1322$ & NM_005544 \\
\hline $1 G F B P 3$ & $\begin{array}{l}\text { Insulin-like growth factor binding } \\
\text { protein } 3\end{array}$ & $\begin{array}{l}\text { F: 5'- ggggtgtacacattcccaac } \\
\text { R: 5'- ggtcatgtccttggcagtct }\end{array}$ & $830-849$ 1080-1061 & NM_000598 \\
\hline BNIP3 & $\begin{array}{l}\text { BCL2/adenovirus E1B 19kDa } \\
\text { interacting protein } 3\end{array}$ & $\begin{array}{l}\text { F: 5’ - ctggacggagtagctccaag } \\
\text { R: } 5 \text { '- gaatattttccggccgactt }\end{array}$ & $368-387589-570$ & NM_004052 \\
\hline$C L U$ & Clusterin & $\begin{array}{l}\text { F: } 5^{\prime} \text { - tcaaaatgctgtcaacgggg } \\
\text { R: 5'- tggtctcattgcacactcct }\end{array}$ & $213-232391-372$ & NM_001831 \\
\hline HK2 & Hexokinase 2 & $\begin{array}{l}\text { F: } 5 \text { '- tctatgccatccctgaggac } \\
\text { R: } 5 \text { - tctctgccttccactccact }\end{array}$ & $786-805$ 1005-986 & NM_000189 \\
\hline PER2 & $\begin{array}{l}\text { Period circadian } \\
\text { regulator } 2\end{array}$ & $\begin{array}{l}\text { F: 5'-cacacagaaggaggagcaga } \\
\text { R: 5'- gatccggtgctctcagatga }\end{array}$ & $2480-2499$ 2719-2700 & NM_022817 \\
\hline $\begin{array}{l}\text { GADD45A } \\
(\text { DDIT1) }\end{array}$ & $\begin{array}{l}\text { Growth arrest and DNA-damage- } \\
\text { inducible alpha } \\
\text { (DNA damage-inducible } \\
\text { transcript-1) }\end{array}$ & $\begin{array}{l}\text { F: 5'-acgaggacgacgacagagat } \\
\text { R: 5'- tcccggcaaaaacaaataag }\end{array}$ & $479-498740-721$ & NM_001924 \\
\hline TPD52 & Tumor protein D52 & $\begin{array}{l}\text { F: 5'- cacagagaccctctcggaag } \\
\text { R: 5'- ccctttggcaatgttctgtt }\end{array}$ & $202-221379-360$ & NM_005079 \\
\hline MKI67 & $\begin{array}{l}\text { Marker of proliferation } \\
\text { Ki- } 67\end{array}$ & $\begin{array}{l}\text { F:5'-ggagaagccccaaccaaaag } \\
\text { R: 5'-taggactaggagctggaggg }\end{array}$ & $2002-20212170-2151$ & NM_002417 \\
\hline ACTB & beta-actin & $\begin{array}{l}\text { F: } 5 \text { '- ggacttcgagcaagagatgg } \\
\text { R: } 5 \text { - agcactgtgttggcgtacag }\end{array}$ & $747-766$ 980-961 & NM_001101 \\
\hline
\end{tabular}


purified from whole serum by protein A affinity column (Phoenix Pharmaceuticals Inc., Belmont, Ca, U.S.A.) was used. The membrane was incubated with anti-NAMPT antibody at 1:1000 dilution for $16 \mathrm{~h}$ at $4^{\circ} \mathrm{C}$. Horseradish peroxidase-conjugated anti-rabbit IgG (Santa Cruz Biotechnology, Santa Cruz, CA, U.S.A.) was used as a secondary antibody diluted 1:5000. The bands were visualized by enhanced chemiluminescence's reagents (Amersham Biosciences). For detection of the beta-actin protein, a rabbit polyclonal anti-beta-actin antibody (ab8227, Abcam, Cambridge, UK) was used. The beta-actin was used to ensure equal loading of the sample.

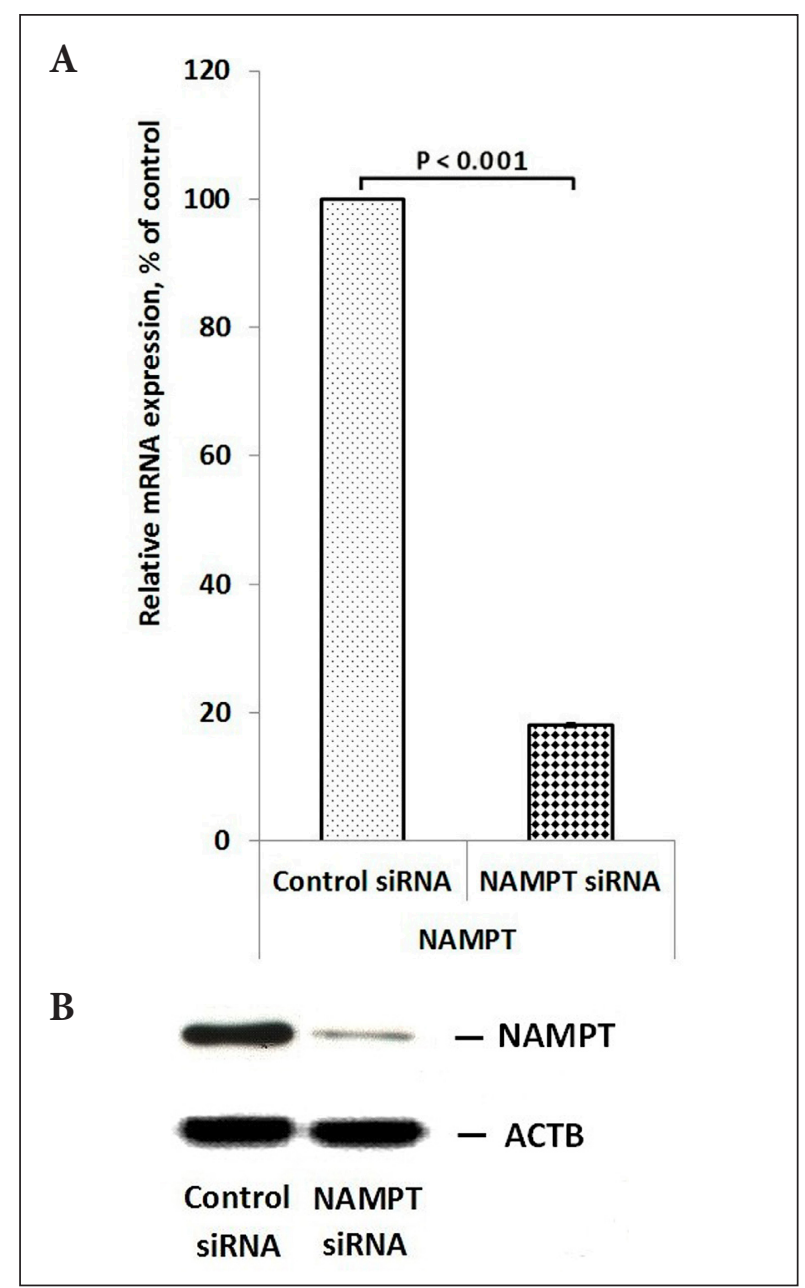

Figure 1. (A) The expression level of nicotinamide phosphoribosyltransferase (NAMPT) mRNA in glioma cells treated by siRNA NAMPT as compared to cells treated by control siRNA. The values of this mRNA expression was normalized to the expression of beta-actin mRNA, expressed as mean \pm SEM and represented as a percent of control $(100 \%) ; n=4$. (B) Western blot analysis of NAMPT protein in glioma cells treated by siRNA NAMPT and control siRNA. The level of ACTB protein was as a marker of protein quantity applied on a gel.

\section{Results}

We analyzed the effect of adipokine NAMPT silencing on the expression of genes encoding IRS1 and some other important polyfunctional proteins in U87 glioma cells.

As shown in Figure 1, the expression level of NAMPT mRNA and protein in U87 glioma cells, transfected by siRNA NAMPT, was strongly downregulated in comparison with the control glioma cells, transfected by control siRNA, 48 hours after transfection. Furthermore, the silencing of NAMPT had smaller but still statistically significant effect $(-24 \%)$ on the proliferation rate of these glioma cells as well as on the expression level of the proliferation marker Ki-67 (-25\%) in comparison with control glioma cells, transfected by control siRNA (Figure 2). Further, we investigated the effect of NAMPT silencing on the expression of gene encoding the insulin receptor substrate 1 , which can interact with insulin receptor and insulin-like growth factor receptors and mediate the control of various cellular processes by insulin as well as insulinlike growth factors, in glioma cells. As shown in Figure 3, the expression level of IRS1 mRNA was strongly up-regulated $(+200 \%)$ in glioma cells after silencing the NAMPT in comparison with the cells, transfected by the control siRNA. More significant up-regulation of the expression level has been shown for gene encoding the insulin-like growth factor binding protein $3(+542 \%)$ in glioma cells with suppressed level of adipokine NAMPT in comparison with control cells (Figure 3). This polyfunctional protein plays an important role in the regulation of the insulin-like growth factors and has additional functions independent of IGFs.

At the same time, the silencing of NAMPT in glioma cells led to a strong down-regulation in the expression level of genes encoding the surviving protein clusterin (CLU) and BCL2/adenovirus E1B 19 $\mathrm{kDa}$ interacting protein 3 (BNIP3): $-96 \%$ and $-82 \%$, correspondingly, in comparison with the control cells (Figure 4). Furthermore, similar but significant less changes in the expression level we received for gene encoding the hexokinase $2(-18 \%)$, in the glioma cells deficient in NAMPT, introduced by NAMPT specific siRNA, in comparison with cells transfected by the control siRNA (Figure 5). Furthermore, as shown in Figurer 5 and Figure 6, the silencing of NAMPT in the glioma cells does not significantly change the expression level of genes encoding the circadian period regulator 2 (PER2), growth arrest, DNA-damage-inducible alpha protein (GADD45A), 

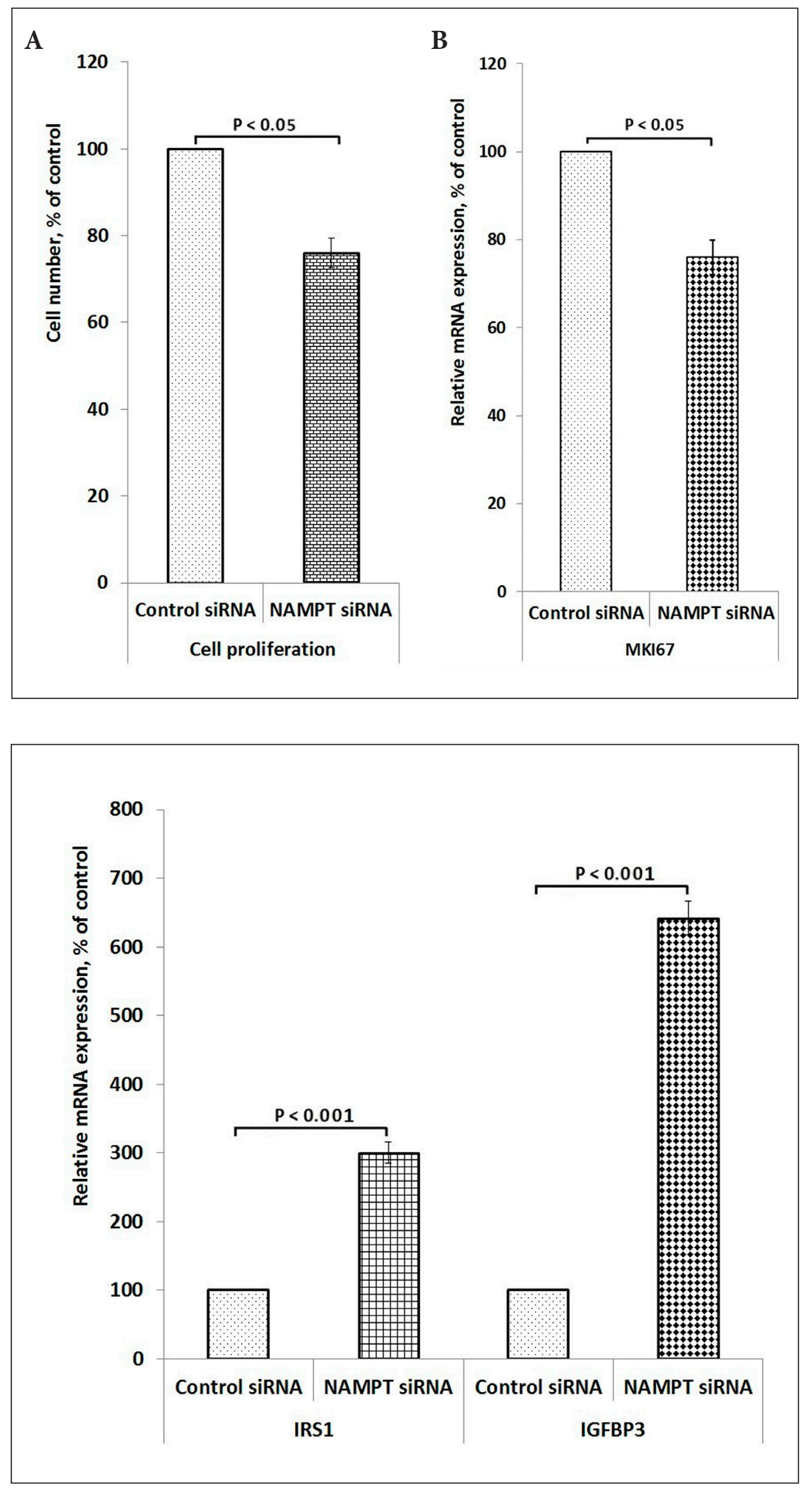

Figure 2. (A) Proliferation rate of glioma cells treated by siRNA NAMPT as compared to cells treated by control siRNA. (B) Effect of NAMPT silencing on the expression of marker of proliferation Ki-67 (MKI67) mRNA in glioma cells treated by siRNA NAMPT and control siRNA. The values of MKI67 mRNA expression was normalized to the expression of beta-actin mRNA, expressed as mean \pm SEM and represented as a percent of control (100\%); $\mathrm{n}=4$.

Figure 3. The expression level of insulin receptor substrate 1 (IRS1) and insulin-like growth factor binding protein 3 (IGFBP3) mRNAs in glioma cells treated by siRNA NAMPT as compared to cells treated by control siRNA. The values of IRS1 and IGFBP3 mRNA expressions were normalized to the expression of beta-actin mRNA, expressed as mean \pm SEM and represented as a percent of control $(100 \%) ; n=4$. 
Figure 4. The expression level of BCL2/adenovirus E1B $19 \mathrm{kDa}$ interacting protein 3 (BNIP3) and clusterin (CLU) mRNAs in glioma cells treated by siRNA NAMPT as compared to cells treated by control siRNA. The values of these mRNA expressions were normalized to the expression of beta-actin mRNA, expressed as mean \pm SEM and represented as a percent of control (100\%); $\mathrm{n}=4$.

Figure 5. Effect of NAMPT silencing on the expression of hexokinase 2 (HK2) and period circadian regulator 2 (PER2) mRNAs in glioma cells treated by siRNA NAMPT and control siRNA. The values of HK2 and PER2 mRNA expressions were normalized to the expression of beta-actin mRNA, expressed as mean \pm SEM and represented as a percent of control (100\%); $\mathrm{n}=4$.
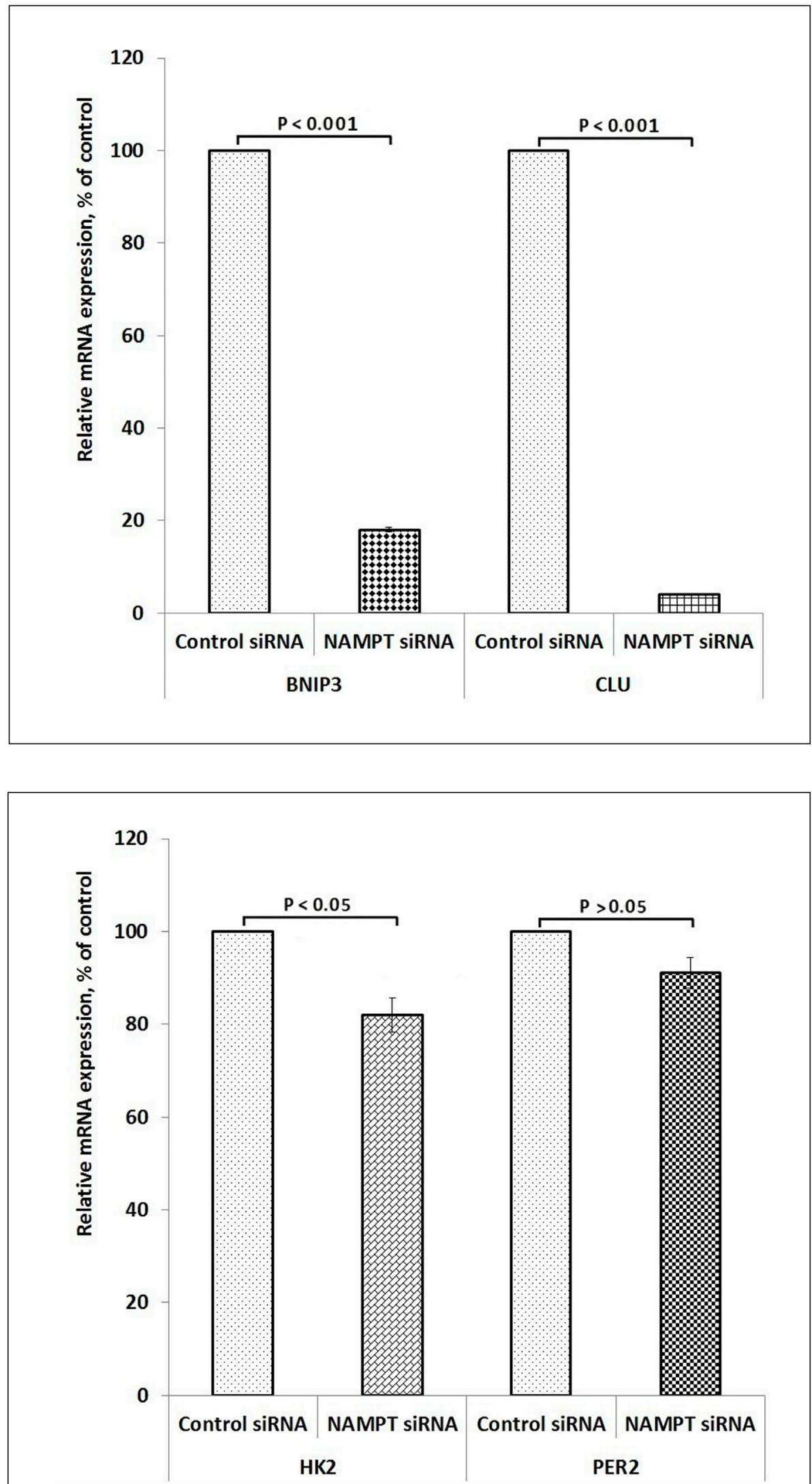
and the tumor protein D52 (TPD52), as compared to the control glioma cells.

By resuming, the silencing of NAMPT, introduced by NAMPT specific siRNA, leads to variable changes in the expression level of genes encoding very important polyfunctional proteins, which play a key role in the regulation of the cell proliferation, surviving, apoptosis, and metabolism, as well as the biological clock.

\section{Discussion}

In this work, we studied the expression of genes encoding the insulin receptor substrate 1, insulinlike growth factor binding protein 3 , and several other genes, which have relation to regulation of the cell proliferation, surviving, apoptosis, and metabolism, in relation to the silencing of NAMPT, introduced by NAMPT specific siRNA. It is important for consideration of a possible importance of adipokine NAMPT in the control of glioma growth through intergenic interactions. There are data indicating that the expression of NAMPT gene is up-regulated in gliomas and that overexpression of this adipokine induces glioma and other cancers growth and that the inhibition of NAMPT by specific inhibitors decreases cancer growth, induces apoptosis, suppresses cell growth, and enhances the susceptibility to oxidative stress as well as sensitizes glioblastoma cells to temozolomide (Minchenko et al. 2015a; Abu Aboud et al. 2016; Feng et al. 2016; Alaee et al. 2017; LucenaCacace et al. 2017; Xu et al. 2017; Zhao et al. 2017; Hesari et al. 2018). We have shown that silencing of NAMPT leads to a strong down-regulation of this adipokine mRNA expression with a subsequent suppression of NAMPT protein level in the glioma cells as well as the suppression of the cell proliferation (Figure 7). Similar results have been received by Bong et al. (2016) using RPMI 8226 cells. They have shown that the silencing of NAMPT decreases the protein level of this adipokine and suppresses the proliferation of these cells. These data agree well with our results concerning the down-regulation of the expression level of Ki-67 marker of proliferation in U87 glioma cells. Accordingly, our data indicating that the silencing of adipokine NAMPT mRNA leads to a significant reduction of this adipokine protein and subsequent suppression of the glioma cell proliferation agree well with the prooncogenic function of NAMPT, which is implicated in cancer development

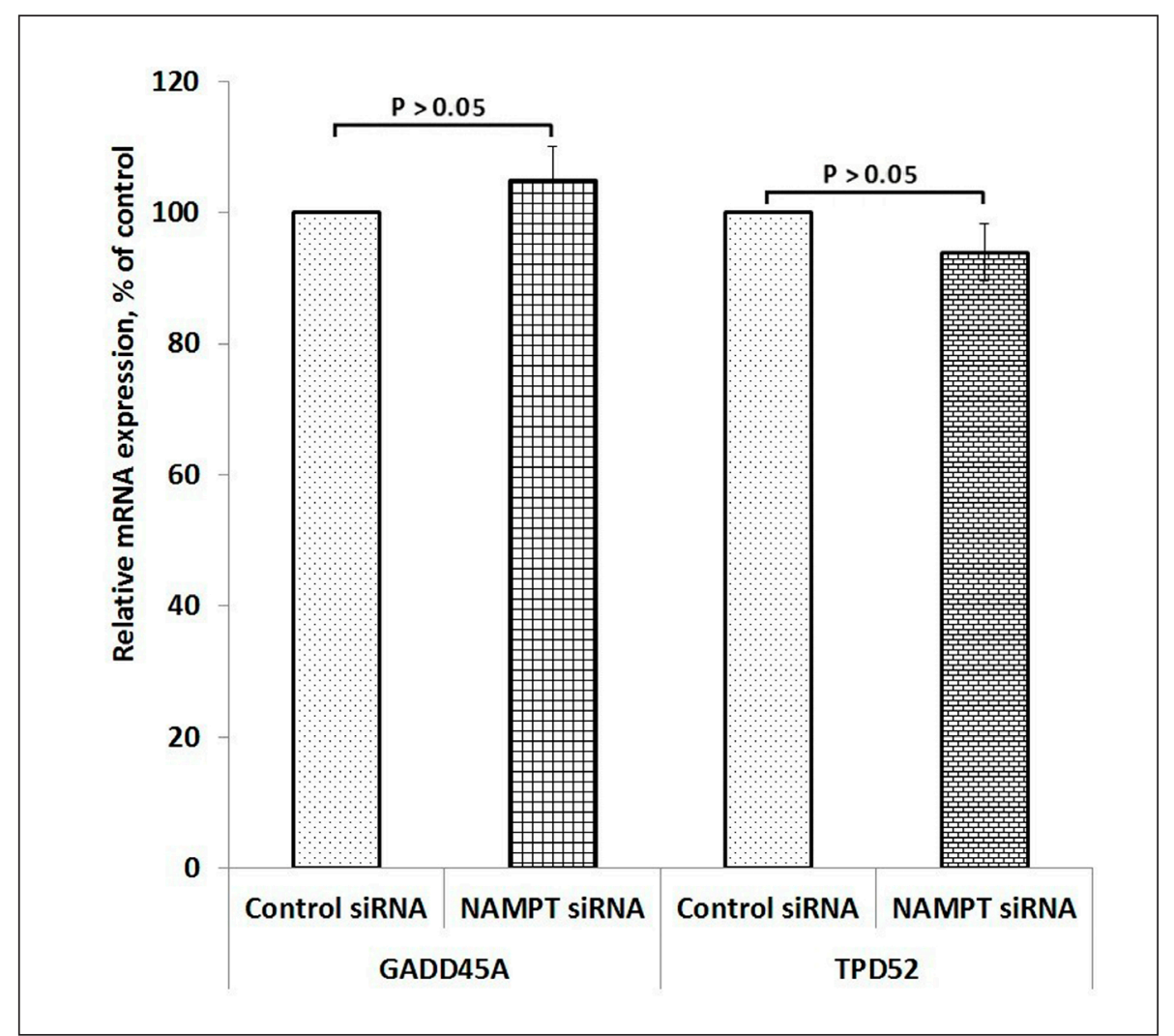

Figure 6. Effect of NAMPT silencing on the expression of growth arrest and DNA-damage-inducible alpha (GADD45A) and tumor protein D52 (TPD52) mRNAs in glioma cells treated by siRNA NAMPT and control siRNA. The values of these mRNA expressions were normalized to the expression of beta-actin mRNA, expressed as mean \pm SEM and represented as a percent of control $(100 \%) ; n=4$. 


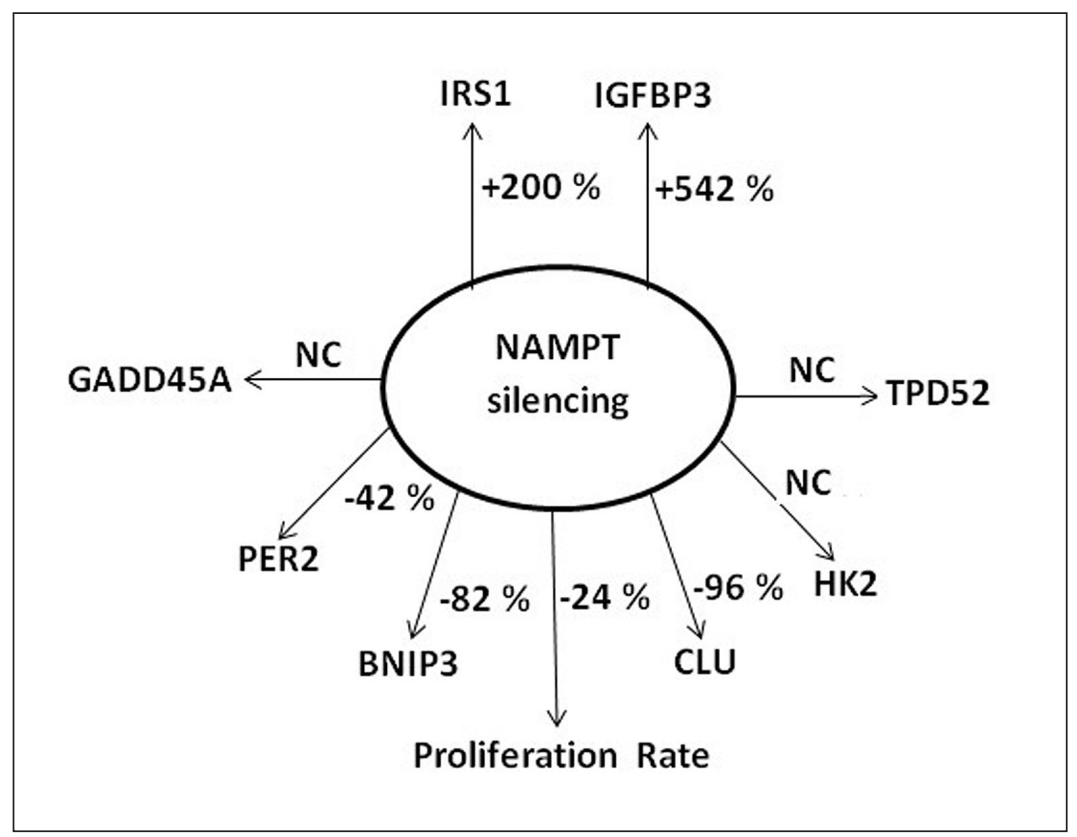

Figure 7. Schematic demonstration of the expression profile of IRS1, IGFBP3, HK2, PER2, BNIP3, CLU, GADD45A, and TPD52 genes as well as cell proliferation rate in glioma cells after NAMPT silencing as compared to control cells treated by control siRNA; NC - no significant changes.

and progression (Minchenko et al. 2015a; Feng et al. 2016; Alaee et al. 2017; Lucena-Cacace et al. 2017; Xu et al. 2017; Zhao et al. 2017; Hesari et al. 2018; Meram et al. 2019).

Previously it has been shown that inhibition of the nicotinamide phosphoribosyltransferase decreases the cell growth and enhances the susceptibility to oxidative stress through down-regulation 325 proteins in the FK866-treated 293T cells as well as through reduction of mitochondrial ribosomal proteins and subsequent mitochondrial dysfunction (Xu et al. 2017). There are also data indicating that NAMPT inhibitor APO866 increased the expression of aggrecan and collagen II inhibited by IL-1beta and the autophagic markers, LC3 and beclin-1 (Shi et al. 2018). These data clearly demonstrate that NAMPT is a pleiotropic protein implicated in the regulation of variable processes as well as in pathogenesis of cancer, diabetes, obesity, and other diseases because it is involved in the basic key cellular functions such as transcription, translation, cell signaling, and metabolism (Zhang et al. 2017) and agree well with our results regarding the dependence of the expression of different genes on the level of NAMPT, which reflects fairly close relationships between different genes in the regulation of their functional activity.

As shown in Figure 7, inducible effect of NAMPT silencing on the expression of gene encoding insulin receptor substrate 1 , which can interact with insulin receptor and insulin-like growth factor receptors and mediate the control of various cellular processes by insulin as well as insulin-like growth factors (Xu et al. 2018; Sun et al. 2019; Wu et al. 2019), is not correlated well with suppression of cell proliferation of glioma cells, but a strong up-regulation of IGFBP3, which has anti-proliferative properties (Yan et al. 2017; Fan et al. 2018; Zhou et al. 2018; Tu et al. 2019), and possibly contributes to the anti-proliferative effect of NAMPT silencing. IGFBP3 is a pleiotropic protein, which plays an important role in the regulation of the insulin-like growth factors and has additional functions independent on IGFs (Ingermann et al. 2010; Nedic et al. 2013; Canel et al. 2017; Wang et al. 2017).

We showed that the silencing of NAMPT adipokine leads to down-regulation of the expression of $H K 2$ and $C L U$ genes in glioma cells (Figure 7). These data agree well with the pro-oncogenic properties of proteins encoded by these genes (Liu et al. 2018; Chen et al. 2014, 2019; Yoo et al. 2019). Actually, there are data indicating that overexpression of HK2 promotes the proliferation and survival of the laryngeal squamous cell carcinoma and that long non-coding RNA PVT1 promotes tumor progression by regulating the miR-143/HK2 axis in the gallbladder cancer (Chen et 
al. 2014, 2019). Moreover, clusterin promotes growth of the renal carcinoma cell by an upregulation of S100A4 gene expression (Liu et al. 2018). At the same time, we showed that the expression of BNIP3 gene is also down-regulated, but BNIP3 has anti-proliferate properties. It is a pleiotropic protein, which plays an important role in the apoptosis and cell proliferation and is down-regulated in tumors (Singh et al. 2018; Shao et al. 2019). There are also data available that low BNIP3 expression is associated with the breast tumor progression, because RNA N6-methyladenosine demethylase FTO promotes this tumor progression through inhibiting BNIP3 (Niu et al. 2019).

The proteins encoding by PER2, GADD45A, and TPD52 genes are also involved in the cancer cell proliferation and tumor growth and are dysregulated in cancer cells (Wang et al. 2016; Xiang et al. 2018; Xiong et al. 2018; Zhang et al. 2018; Hu et al. 2019; Rahman et al. 2019). At the same time, the expression of these genes was resistant to the silencing of adipokine NAMPT in the glioma cells. It is possible that PER2, GADD45A, and TPD52 genes are not included in the gene network associated with the adipokine NAMPT and the silencing of this adipokine did not change their expression profile.
This study provides unique insights into the molecular mechanisms regulating the expression of genes encoding IRS1, IGFBP3, HK2, PER2, CLU, BNIP3, TPD52, GADD45A, and MKI67 proteins in glioma cells in response to silencing of adipokine NAMPT and their correlation with reduced cell proliferation, attesting to the fact that this adipokine is a necessary component of malignant tumor growth and cell survival. It is possible that there is functional gene network and that knockdown one gene disruptions genome stability and leads to numerous changes in gene expression directly or indirectly initiated by the excluded gene, in particular NAMPT. However, the detailed molecular mechanisms of this regulation have not been yet clearly defined and warrant further investigation.

\section{Acknowledgments}

The authors thank Prof. Michel Moenner (University Bordeaux 1, France) for his interest and support of this work. This work was funded by the State Budget Program "Support for the Development of Priority Areas of Scientific Research" (Code: 6541230).

\section{References}

Abu Aboud O, Chen CH, Senapedis W, Baloglu E, Argueta C, Weiss RH. Dual and specific inhibition of NAMPT and PAK4 by KPT-9274 decreases kidney cancer growth. Mol Cancer Ther 15, 2119-2129, 2016.

Alaee M, Khaghani S, Behroozfar K, Hesari Z, Ghorbanhosseini SS, Nourbakhsh M. Inhibition of ncotinamide phosphoribosyltransferase induces apoptosis in estrogen receptor-positive MCF-7 breast cancer cells. J Breast Cancer 20, 20-26, 2017.

Annie L, Gurusubramanian G, Roy VK. Estrogen and progesterone dependent expression of visfatin/NAMPT regulates proliferation and apoptosis in mice uterus during estrous cycle. J Steroid Biochem Mol Biol 185, 225236, 2019.

Armstead VE, Minchenko AG, Campbell B, Lefer AM. P-selectin is up-regulated in vital organs during murine traumatic shock. FASEB J 11, 1271-1279, 1997.

Bong IP, Ng CC, Fakiruddin SK, Lim MN, Zakaria Z. Small interfering RNA-mediated silencing of nicotinamide phosphoribosyltransferase (NAMPT) and lysosomal trafficking regulator (LYST) induce growth inhibition and apoptosis in human multiple myeloma cells: A preliminary study. Bosn J Basic Med Sci 16, 268-275, 2016.

Canel M, Byron A, Sims AH, Cartier J, Patel H, Frame MC, Brunton VG, Serrels B, Serrels A. Nuclear FAK and Runx1 cooperate to regulate IGFBP3, cell-cycle progression, and tumor growth. Cancer Res 77, 5301-5312, 2017.

Carbone F, Liberale L, Bonaventura A, Vecchie A, Casula M, Cea M, Monacelli F, Caffa I, Bruzzone S, Montecucco F, Nencioni A. Regulation and function of extracellular nicotinamide phosphoribosyltransferase/visfatin. Compr Physiol 7, 603-662, 2017.

Cheleschi S, Giordano N, Volpi N, Tenti S, Gallo I, Di Meglio M, Giannotti S, Fioravanti A. A Complex Relationship between Visfatin and Resistin and microRNA: An in vitro study on human chondrocyte cultures. Int J Mol Sci 19, E3909, 2018.

Chen J, Zhang S, Li Y, Tang Z, Kong W. Hexokinase 2 overexpression promotes the proliferation and survival of laryngeal squamous cell carcinoma. Tumour Biol 35, 3743-3753, 2014. 
Chen J, Yu Y, Li H, Hu Q, Chen X, He Y, Xue C, Ren F, Ren Z, Li J, Liu L, Duan Z, Cui G, Sun R. Long non-coding RNA PVT1 promotes tumor progression by regulating the miR-143/HK2 axis in gallbladder cancer. Mol Cancer 18, 33, 2019.

Fan X, Wang Y, Jiang T, Cai W, Jin Y, Niu Y, Zhu H, Bu Y. B-Myb mediates proliferation and migration of non-smallcell lung cancer via suppressing IGFBP3. Int J Mol Sci 19, E1479, 2018.

Feng J, Yan PF, Zhao HY, Zhang FC, Zhao WH, Feng M. Inhibitor of nicotinamide phosphoribosyltransferase sensitizes glioblastoma cells to temozolomide via activating ROS/JNK signaling pathway. Biomed Res Int 2016, $1450843,2016$.

Flehmig G, Scholz M, Kloting N, Fasshauer M, Tonjes A, Stumvoll M, Youn BS, Bluher M. Identification of adipokine clusters related to parameters of fat mass, insulin sensitivity and inflammation. PLoS One 9, e99785, 2014.

Goktas Z, Owens S, Boylan M, Syn D, Shen CL, Reed DB, San Francisco S, Wang S. Associations between tissue visfatin/nicotinamide, phosphoribosyltransferase (Nampt), retinol binding protein-4, and vaspin concentrations and insulin resistance in morbidly obese subjects. Mediators Inflamm 2013, 861496, 2013.

Hesari Z, Nourbakhsh M, Hosseinkhani S, Abdolvahabi Z, Alipour M, Tavakoli-Yaraki M, Ghorbanhosseini SS, Yousefi Z, Jafarzadeh M, Yarahmadi S. Down-regulation of NAMPT expression by mir-206 reduces cell survival of breast cancer cells. Gene 673, 149-158, 2018.

Hu Y, Jin R, Gao M, Xu H, Zou S, Li X, Xing C, Wang Q, Wang H, Feng J, Hu M, Song L. Transcriptional repression of IKKbeta by p53 in arsenite-induced GADD45alpha accumulation and apoptosis. Oncogene 38, 731-746, 2019.

Huang Z, Tian G, Cheng S, Zhao D, Zhang Y, Jia Y, Zhou F. Polydatin attenuates atherosclerosis in ApoE ${ }^{-/-} \mathrm{mice}^{-}$ through PBEF mediated reduction of cholesterol deposition. Am J Chin Med 46, 1841-1859, 2018.

Ingermann AR, Yang YF, Han J, Mikami A, Garza AE, Mohanraj L, Fan L, Idowu M, Ware JL, Kim HS, Lee DY, Oh Y. Identification of a novel cell death receptor mediating IGFBP-3-induced anti-tumor effects in breast and prostate cancer. J Biol Chem 285, 30233-30246, 2010.

Li H, Bai E, Zhang Y, Jia Z, He S, Fu J. Role of nampt and visceral adiposity in esophagogastric junction adenocarcinoma. J Immunol Res 2017, 3970605, 2017.

Li H, Yu L, Zhao C. Dioscin attenuates high-fat diet-induced insulin resistance of adipose tissue through the IRS-1/ PI3K/Akt signaling pathway. Mol Med Rep 19, 1230-1237, 2019.

Liang N, Chen Y, Yang L, He S, Liu T. Visfatin increases miR-21 to promote migration in HCC. Cell Mol Biol (Noisyle-grand) 64, 48-52, 2018.

Liu Y, Men C, Xu Y, Zhao K, Luo L, Dong D, Yu Q. Clusterin promotes growth and invasion of clear cell renal carcinoma cell by upregulation of S100A4 expression. Cancer Biomark 21, 915-923, 2018.

Lucena-Cacace A, Otero-Albiol D, Jimenez-Garcia MP, Peinado-Serrano J, Carnero A. NAMPT overexpression induces cancer stemness and defines a novel tumor signature for glioma prognosis. Oncotarget 8, 99514-99530, 2017.

Meram AT, Alzubaidi Y, Cotelingam J, Ghali G, Lopez L, Coppola D, Shackelford R. Nicotinamide phosphoribosyl transferase is increased in osteosarcomas and chondrosarcomas compared to benign bone and cartilage. Anticancer Res 39, 1761-1765, 2019.

Minchenko OH, Opentanova IL, Minchenko DO, Ogura T, Esumi H. Hypoxia induces transcription of 6-phosphofructo-2-kinase/fructose-2,6-bisphosphatase 4 gene via hypoxia-inducible factor-1alpha activation. FEBS Lett 576, 14-20, 2004.

Minchenko DO, Novik YE, Maslak HS, Tiazhka OV, Minchenko OH. Expression of PFKFB, HK2, NAMPT, TSPAN13 and HSPB8 genes in pediatric glioma. Lik Sprava 7-8, 43-48, 2015a.

Minchenko OH, Tsymbal DO, Minchenko DO, Moenner M, Kovalevska OV, Lypova NM. Inhibition of kinase and endoribonuclease activity of ERN1/IRE1 affects expression of proliferation-related genes in U87 glioma cells. Endoplasm Reticul Stress Dis 2, 18-29, 2015 b.

Nedic O, Robajac D, Sunderic M, Miljus G, Dukanovic B, Malenkovic V. Detection and identification of oxidized insulin-like growth factor-binding proteins and receptors in patients with colorectal carcinoma. Free Radic Biol Med 65, 1195-1200, 2013.

Nielsen KN, Peics J, Ma T, Karavaeva I, Dall M, Chubanava S, Basse AL, Dmytriyeva O, Treebak JT, Gerhart-Hines Z. NAMPT-mediated $\mathrm{NAD}^{+}$biosynthesis is indispensable for adipose tissue plasticity and development of obesity. Mol Metab 11, 178-188, 2018.

Niu Y, Lin Z, Wan A, Chen H, Liang H, Sun L, Wang Y, Li X, Xiong XF, Wei B, Wu X, Wan G. RNA N6-methyladenosine demethylase FTO promotes breast tumor progression through inhibiting BNIP3. Mol Cancer 18, 46, 2019. 
Ohanna M, Cerezo M, Nottet N, Bille K, Didier R, Beranger G, Mograbi B, Rocchi S, Yvan-Charvet L, Ballotti R, Bertolotto C. Pivotal role of NAMPT in the switch of melanoma cells toward an invasive and drug-resistant phenotype. Genes Dev 32, 448-461, 2018.

Olszanecka-Glinianowicz M, Owczarek A, Bozentowicz-Wikarek M, Brzozowska A, Mossakowska M, Zdrojewski T, Grodzicki T, Wiecek A, Chudek J. Relationship between circulating visfatin/NAMPT, nutritional status and insulin resistance in an elderly population - results from the PolSenior substudy. Metabolism 63, 1409-1418, 2014.

Poljsak B. NAMPT-mediated NAD biosynthesis as the internal timing mechanism: In NAD+ world, time is running in its own way. Rejuvenation Res 21, 210-224, 2018.

Qu M, Duffy T, Hirota T, Kay SA. Nuclear receptor HNF4A transrepresses CLOCK:BMAL1 and modulates tissuespecific circadian networks. Proc Natl Acad Sci USA 115, E12305-E12312, 2018.

Rahman S, Kraljevic Pavelic S, Markova-Car E. Circadian (de)regulation in head and neck squamous cell carcinoma. Int J Mol Sci 20, E2662, 2019.

Shackelford RE, Mayhall K, Maxwell NM, Kandil E, Coppola D. Nicotinamide phosphoribosyltransferase in malignancy: a review. Genes Cancer 4, 447-56, 2013.

Shao Y, Liu Z, Liu J, Wang H, Huang L, Lin T, Liu J, Wei Q, Zeng H, He G, Li X. Expression and epigenetic regulatory mechanism of BNIP3 in clear cell renal cell carcinoma. Int J Oncol 54, 348-360, 2019.

Singh A, Azad M, Shymko MD, Henson ES, Katyal S, Eisenstat DD, Gibson SB. The BH3 only Bcl-2 family member BNIP3 regulates cellular proliferation. PLoS ONE 13, e0204792, 2018.

Shi C, Wu H, Du D, Im HJ, Zhang Y, Hu B, Chen H, Wang X, Liu Y, Cao P, Tian Y, Shen X, Gao R, van Wijnen AJ, Ye X, Yuan W. Nicotinamide phosphoribosyltransferase inhibitor APO866 prevents IL-1beta-induced human nucleus pulposus cell degeneration via autophagy. Cell Physiol Biochem 49, 2463-2482, 2018.

Sun Y, Zhou J, Shi L, Li J, Chen J. MicroRNA-466 inhibits cell proliferation and invasion in osteosarcoma by directly targeting insulin receptor substrate 1. Mol Med Rep 19, 3345-3352, 2019.

Tabassum A, Zaidi SNF, Yasmeen K, Mahboob T. Potential role of peroxisome proliferator activated receptor gamma activation on serum visfatin and trace elements in high fat diet induced type 2 diabetes mellitus. Life Sci 205, 164-175, 2018.

Tu W, Yang B, Leng X, Pei X, Xu J, Liu M, Dong Q, Tao D, Lu Y, Liu Y, Yang Y. Testis-specific protein, Y-linked 1 activates PI3K/AKT and RAS signaling pathways through suppressing IGFBP3 expression during tumor progression. Cancer Sci 110, 1573-1586, 2019.

Wang HH, Wang YC, Wu DW, Hung CS, Chen CY, Lee H. Targeting insulin-like growth factor-binding protein-3 by microRNA-125b promotes tumor invasion and poor outcomes in non-small-cell lung cancer. Tumour Biol 39, 1010428317694316, 2017.

Wang Y, Chen CL, Pan QZ, Wu YY, Zhao JJ, Jiang SS, Chao J, Zhang XF, Zhang HX, Zhou ZQ, Tang Y, Huang XQ, Zhang JH, Xia JC. Decreased TPD52 expression is associated with poor prognosis in primary hepatocellular carcinoma. Oncotarget 7, 6323-6334, 2016.

Wu L, Li Y, Li J, Ma D. MicroRNA-664 targets insulin receptor substrate 1 to suppress cell proliferation and invasion in breast cancer. Oncol Res 27, 459-467, 2019.

Xiang R, Cui Y, Wang Y, Xie T, Yang X, Wang Z, Li J, Li Q. Circadian clock gene Per2 downregulation in nonsmall cell lung cancer is associated with tumour progression and metastasis. Oncol Rep 40, 3040-3048, 2018.

Xiong H, Yang Y, Yang K, Zhao D, Tang H, Ran X. Loss of the clock gene PER2 is associated with cancer development and altered expression of important tumor-related genes in oral cancer. Int J Oncol 52, 279-287, 2018.

Xu H, Lee MS, Tsai PY, Adler AS, Curry NL, Challa S, Freinkman E, Hitchcock DS, Copps KD, White MF, Bronson RT, Marcotrigiano M, Wu Y, Clish CB, Kalaany NY. Ablation of insulin receptor substrates 1 and 2 suppresses Kras-driven lung tumorigenesis. Proc Natl Acad Sci USA 115, 4228-4233, 2018.

Xu R, Yuan Z, Yang L, Li L, Li D, Lv C. Inhibition of NAMPT decreases cell growth and enhances susceptibility to oxidative stress. Oncol Rep 38, 1767-1773, 2017.

Yan J, Yang X, Li L, Liu P, Wu H, Liu Z, Li Q, Liao G, Wang X. Low expression levels of insulin-like growth factor binding protein-3 are correlated with poor prognosis for patients with hepatocellular carcinoma. Oncol Lett 13, 3395-3402, 2017.

Yoo JJ, Yu SJ, Na J, Kim K, Cho YY, Lee YB, Cho EJ, Lee JH, Kim YJ, Youn H, Yoon JH. Hexokinase-II inhibition synergistically augments the anti-tumor efficacy of sorafenib in hepatocellular carcinoma. Int J Mol Sci 20, E1292, 2019.

Zhao H, Tang W, Chen X, Wang S, Wang X, Xu H, Li L. The NAMPT/E2F2/SIRT1 axis promotes proliferation and inhibits p53-dependent apoptosis in human melanoma cells. Biochem Biophys Res Commun 493, 77-84, 2017. 
Zhang LQ, Van Haandel L, Xiong M, Huang P, Heruth DP, Bi C, Gaedigk R, Jiang X, Li DY, Wyckoff G, Grigoryev DN, Gao L, Li L, Wu M, Leeder JS, Ye SQ. Metabolic and molecular insights into an essential role of nicotinamide phosphoribosyltransferase. Cell Death Dis 8, e2705, 2017.

Zhang Y, Li Y, Wang J, Lei P. Long noncoding RNA ferritin heavy polypeptide 1 pseudogene 3 controls glioma cell proliferation and apoptosis via regulation of the microRNA2245p/tumor protein D52 axis. Mol Med Rep 18, 4239-4246, 2018.

Zhou N, Sun Z, Li N, Ge Y, Zhou J, Han Q, Zhao L, Bai C. miR197 promotes the invasion and migration of colorectal cancer by targeting insulin-like growth factor-binding protein 3. Oncol Rep 40, 2710-2721, 2018. 